\title{
Radiative Penguin Decays at BABAR
}

\author{
Anders Ryd representing the BABAR collaboration \\ California Institute of Technology, 356-48 Caltech, Pasadena, CA 91125, USA
}

\begin{abstract}
We report preliminary results based on a data sample of $20.7 \mathrm{fb}^{-1}$ recorded at the $\Upsilon(4 S)$ resonance by the BABAR detector at the PEP-II energy asymmetric collider at the Stanford Linear Accelerator Center. We have measured the branching fraction $\mathcal{B}\left(B^{0} \rightarrow K^{* 0} \gamma\right)=(4.39 \pm 0.41 \pm$ $0.27) \times 10^{-5}$ and measured a charge asymmetry in the $B \rightarrow K^{*} \gamma$ decays consistent with zero: $A_{C P}=-0.035 \pm 0.076 \pm 0.012$. We also searched for the decay $B^{0} \rightarrow \gamma \gamma$ and placed the $90 \%$ C.L. limit $\mathcal{B}\left(B^{0} \rightarrow \gamma \gamma\right)<1.7 \times 10^{-6}$. The search for the electroweak penguin decays $B \rightarrow K^{(*)} \ell^{+} \ell^{-}$ yielded the limits $\mathcal{B}\left(B \rightarrow K \ell^{+} \ell^{-}\right)<0.6 \times 10^{-6}$ and $\mathcal{B}\left(B \rightarrow K^{*} \ell^{+} \ell^{-}\right)<2.5 \times 10^{-6}$ at the $90 \%$ C.L.
\end{abstract}

\section{INTRODUCTION}

The decays $B \rightarrow K^{*} \gamma, B \rightarrow K^{(*)} \ell^{+} \ell^{-}$, and $B^{0} \rightarrow \gamma \gamma$, reported on here, are examples of decays taking place via a flavor-changing neutral current (FCNC). In the Standard Model, FCNC transitions are forbidden at tree level and only takes place at higher order in amplitudes involving loops. An example of a quark level diagrams for a FCNC transition is shown in Figure 1 for the $b \rightarrow s \gamma$ transition. The decay $B \rightarrow K^{*} \gamma$, observed by CLEO [1], was the first observation of an electro-magnetic penguin decay of a $B$ meson. CLEO also observed the inclusive decay $b \rightarrow s \gamma[2]$.

As these decays are suppressed in the Standard Model they provide an interesting window to look for physics beyond the Standard Model. New, heavy, particles as predicted e.g. by sypersymmetry can contribute to the rate of these decays by appearing virtually in the loop diagrams. The decay $B \rightarrow K^{*} \ell^{+} \ell^{-}$is interesting as new physics can affect not only the rate, but also kinematic distributions, which in some cases can be predicted with less model dependence than the rate and allow for stringent tests of the Standard Model. For example, the lepton forward-backward asymmetry is expected to have a zero at $q^{2}=m_{\ell^{+} \ell^{-}}^{2} \approx 3.0 \mathrm{GeV}^{2} / c^{4}$, see $e . g$. Ref. [3].

In all of the analysis reported here we make use of the standard $B$ reconstruction variables $\Delta E=\sum_{i} E_{i}^{*}-E_{\text {beam }}^{*}$ and $m_{\mathrm{ES}}=\sqrt{E_{\text {beam }}^{* 2}-\left|\sum_{i} \mathbf{p}^{*}{ }_{i}\right|^{2}}$, where a ${ }^{*}$ denotes a quantity measured in the $e^{+} e^{-}$collision rest frame (CM), $E_{i}$ and $\mathbf{p}_{i}$ are the energy and momentum respectively of the $i$ th daughter of the $B$ candidate, and $E_{\text {beam }}^{*}$ is the beam energy in the the $e^{+} e^{-}$rest frame. $\Delta E$ peaks at zero for signal events and $m_{\mathrm{ES}}$ peaks at the $B$ mass.

CP618, Heavy Flavor Physics: Ninth International Symposium, edited by A. Ryd and F. C. Porter () 2002 American Institute of Physics 0-7354-0064-4/02/\$19.00

143 


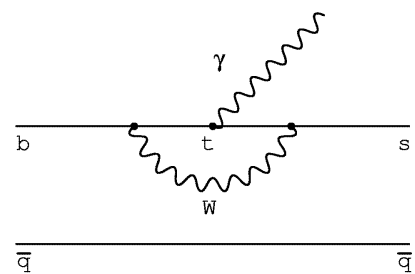

FIGURE 1. Quark level diagram contributing to $b \rightarrow s \gamma$.

\section{DATA SAMPLE}

The data sample used in these analyses was recorded with the BABAR detector from October 1999 to October 2000. Most of this time PEP-II ran on the $\Upsilon(4 S)$ resonance and we recorded $20.7 \mathrm{fb}^{-1}$ on the resonance. We also collected a $2.6 \mathrm{fb}^{-1}$ sample at a center-of-mass energy $40 \mathrm{MeV}$ below the $\Upsilon(4 S)$ resonance. The off-resonance sample is useful for modeling non- $B \bar{B}$ backgrounds, in particular non-resonant $q \bar{q}$ production. The on-resonance sample corresponds to $(22.7 \pm 0.4) \times 10^{6} B \bar{B}$ pairs. The BABAR detector is described in detail in Ref. [4].

\section{STUDIES OF $B \rightarrow K^{*} \gamma$}

We have reconstructed the decays $B^{+} \rightarrow K^{*+} \gamma$ and $B^{0} \rightarrow K^{* 0} \gamma$ with $K^{*+} \rightarrow K_{S}^{0} \pi^{+}, K^{+} \pi^{0}$ and $K^{* 0} \rightarrow K_{S}^{0} \pi^{0}, K^{+} \pi^{-}$. Events are selected that have a photon candidate with a CM energy between 2.30 and $2.85 \mathrm{GeV}$. Photon candidates are vetoed if combined with any other photon candidate in the event give a mass within $2 \sigma$ of either the $\pi^{0}$ or $\eta$ mass.

The photon candidate is combined with a $K^{*}$ candidate to form the $B$ candidate. The $K^{*}$ candidate is required to have an invariant mass, $m_{K \pi}$, within $100 \mathrm{MeV} / c^{2}$ of the mean $K^{*}(892)$ mass. For the $B$ candidate we calculate $\Delta E \equiv E_{K^{*}}^{*}+E_{\gamma}^{*}-E_{\text {beam }}^{*}$. We require that $-200<\Delta E<100 \mathrm{MeV}$ for modes without a $\pi^{0}$ and $-225<\Delta E<125 \mathrm{MeV}$ for modes with a $\pi^{0}$. The signal yields are extracted in $m_{\mathrm{ES}}$. For the modes without a $\pi^{0}$ the magnitude of the photon momentum, $\left|p_{\gamma}^{*}\right|=E_{\gamma}^{*}$, is rescaled such that $\Delta E=0$ when calculating $m_{\mathrm{ES}}$. This rescaling removes the low tail in $m_{\mathrm{ES}}$ due to energy leakage in the calorimeter.

The main background in the analysis comes from non-resonant $q \bar{q}$ production. Most commonly, photons are either from initial state radiation or from decays of $\pi^{0}$ and $\eta$ mesons. This background is suppressed by cutting on three variables that separate the background from the signal. The most powerful cut is $\left|\cos \theta_{\mathrm{T}}^{*}\right|<0.8$, where $\theta_{\mathrm{T}}^{*}$ is the angle between the photon candidate and the thrust axis of the rest of the event, excluding the $K^{*}$ daughter particles. The distribution of $\left|\cos \theta_{\mathrm{T}}^{*}\right|$ is shown in Figure 2. We also require $\left|\cos \theta_{\mathrm{H}}\right|<0.75$, where $\theta_{\mathrm{H}}$ is the decay angle of the kaon in the $K^{*}$ rest frame. As the produced $K^{*}$ mesons in the signal mode have either helicity \pm 1 , but not 0 , the signal distribution in $\theta_{\mathrm{H}}$ is given by a $\sin ^{2} \theta_{\mathrm{H}}$ distribution, whereas the background is approximately flat in $\left|\cos \theta_{\mathrm{H}}\right|$. Similarly, we also require $\left|\cos \theta_{B}\right|<0.75$, where $\theta_{B}$ is 


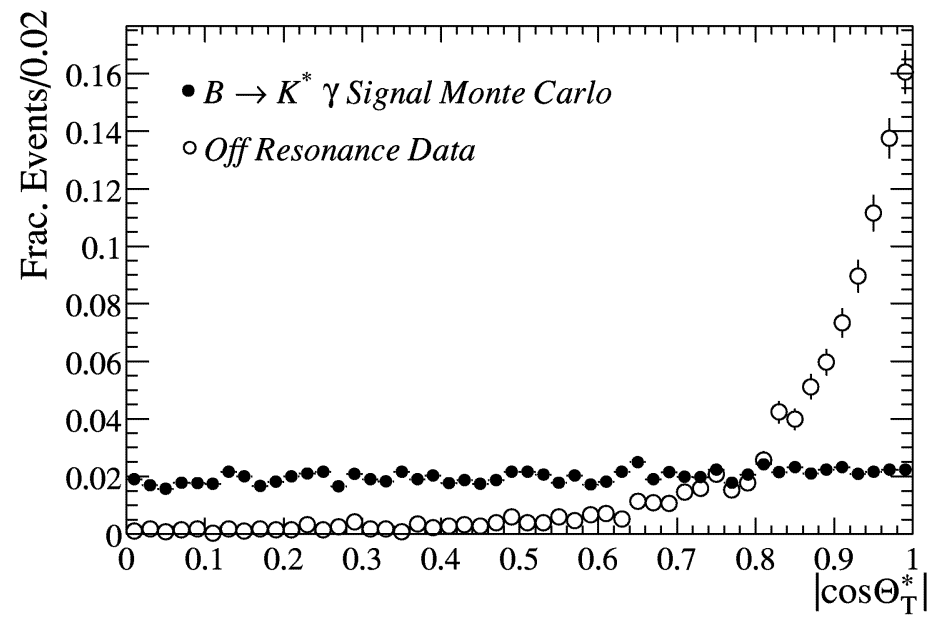

FIGURE 2. Distribution of $\left|\cos \theta_{\mathrm{T}}^{*}\right|$ for signal (solid dots) and off resonance data (open circles).

TABLE 1. The table summarizes efficiency, including $K^{*}$ branching fractions, $(\varepsilon \times \mathcal{B})$; signal yields; cross feed background; and the measured branching fraction.

\begin{tabular}{lllll}
\hline Mode & $\varepsilon \times \mathcal{B}(\%)$ & Signal yield & Cross feed & $\mathcal{B}\left(B \rightarrow K^{*} \gamma\right) / 10^{-5}$ \\
\hline$K^{+} \pi^{-}$ & 14.1 & $139.2 \pm 13.1$ & 1 & $4.39 \pm 0.41 \pm 0.27$ \\
$K^{+} \pi^{0}$ & 5.1 & $57.6 \pm 10.4$ & 3.8 & $5.52 \pm 1.07 \pm 0.33$ \\
$K_{S}^{0} \pi^{0}$ & 1.4 & $14.8 \pm 5.6$ & 1.4 & $4.10 \pm 1.71 \pm 0.42$ \\
$K_{S}^{0} \pi^{+}$ & 2.9 & $28.4 \pm 6.4$ & 1.7 & $3.12 \pm 0.76 \pm 0.21$ \\
\hline
\end{tabular}

the decay angle of the $B$ candidate in the $\Upsilon(4 S)$ frame.

In the four modes studied we obtain the signals as shown in Figure 3, and the results are summarized in Table 1. We also show the $\Delta E$ and $m_{K \pi}$ distributions in Figure 4, which shows that the signal is consistent with $B \rightarrow K^{*}(892) \gamma$. The largest systematic errors come from the photon detection efficiency and tracking efficiency. Recent predictions, Refs. [5] and [6], based on NLO calculations predicts somewhat larger branching fractions $\mathcal{B}\left(B \rightarrow K^{*} \gamma\right)=\left(7.1_{-2.3}^{+2.5}\right) \times 10^{-5}$ and $\mathcal{B}\left(B \rightarrow K^{*} \gamma\right)=\left(7.9_{-3.0}^{+3.5}\right) \times 10^{-5}$, respectively. The theoretical uncertainty is dominated by the $B \rightarrow K^{*}$ form factor.

Using the charge of either the $K$ or $K^{*}$ we measure the charge asymmetry,

$$
A_{C P} \equiv \frac{\Gamma\left(\bar{B} \rightarrow \bar{K}^{*} \gamma\right)-\Gamma\left(B \rightarrow K^{*} \gamma\right)}{\Gamma\left(\bar{B} \rightarrow \bar{K}^{*} \gamma\right)+\Gamma\left(B \rightarrow K^{*} \gamma\right)}
$$

and find $A_{C P}=-0.035 \pm 0.076 \pm 0.012$, consistent with the expectation of a rather small asymmetry (about $-0.5 \%$ ) in the Standard Model [5]. 


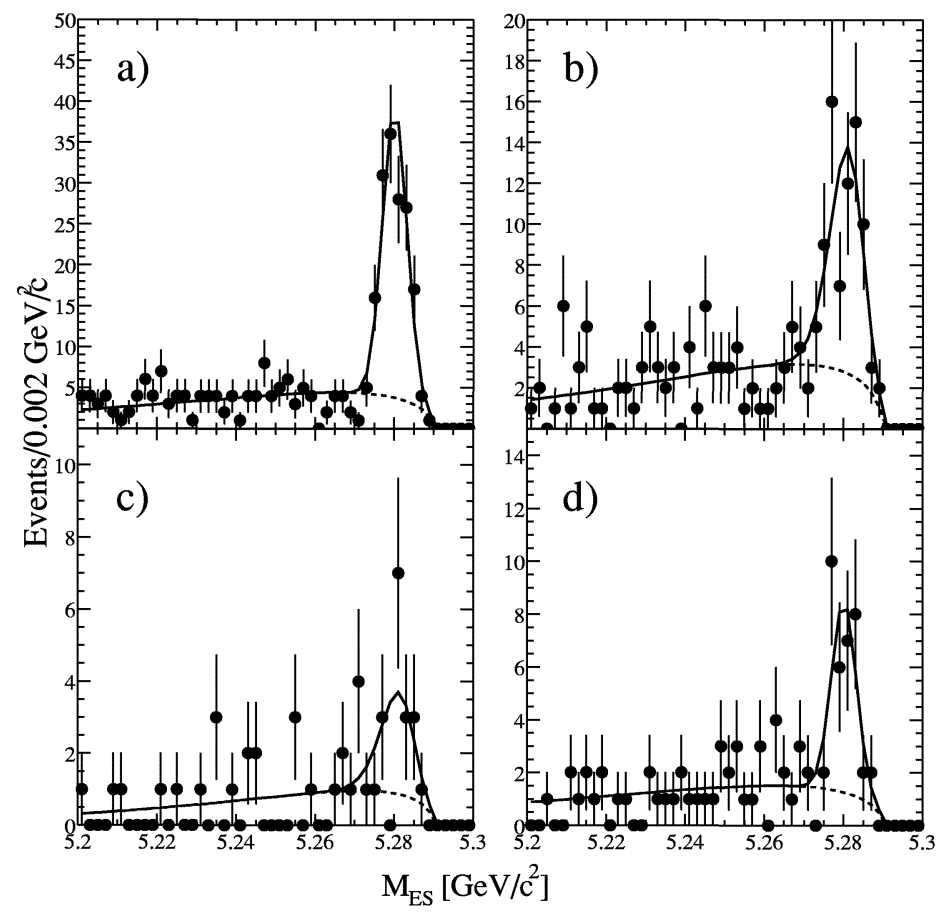

FIGURE 3. Signals in the $B \rightarrow K^{*} \gamma$ analysis where the $K^{*}$ is reconstructed in the modes a) $K^{+} \pi^{-}$, b) $K^{+} \pi^{0}$, c) $K_{S}^{0} \pi^{0}$, and d) $K_{S}^{0} \pi^{+}$.
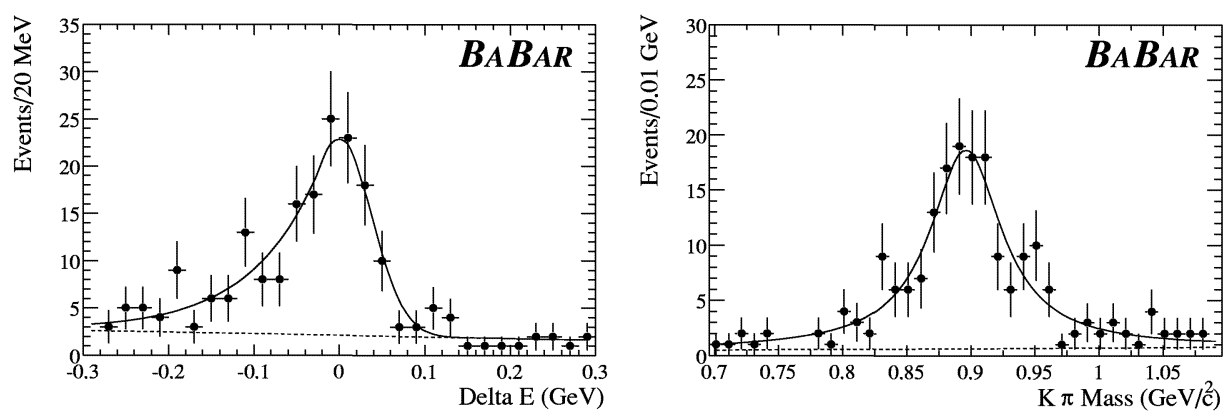

FIGURE 4. The $\Delta E$ (left) and $m_{K \pi}$ (right) distributions in the $K^{+} \pi^{-}$mode in the $B \rightarrow K^{*} \gamma$ analysis.

\section{SEARCH FOR $B^{0} \rightarrow \gamma \gamma$}

The decay $B^{0} \rightarrow \gamma \gamma$ is highly suppressed in the Standard Model, expected branching fractions are $(0.1-2) \times 10^{-8}$ [7]. Figure 5 shows diagrams that contributes to the $B^{0} \rightarrow \gamma \gamma$ decay. 

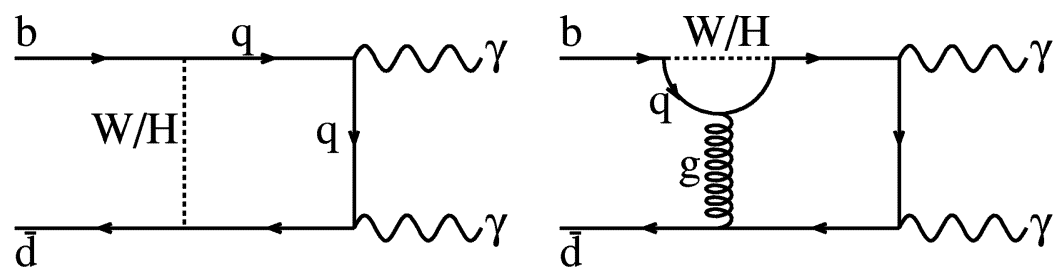

FIGURE 5. Quark level diagrams contributing to the $B^{0} \rightarrow \gamma \gamma$ process.

The search for $B^{0} \rightarrow \gamma \gamma$ was done blind, the signal region in $\Delta E$ versus $m_{\mathrm{ES}}$ were blind during the process of optimizing the event selection criteria. After unblinding, the signal yield is extracted by counting the number of events in the signal region. The data sample used in this analysis is somewhat smaller, $18 \mathrm{fb}^{-1}$, than what is used in the other two analysis described in this presentation. Photon candidates in this analysis are rejected if they combined with any other photon candidate in the event are within $3 \sigma$ of either the $\pi^{0}$ or $\eta$ mass. This cut is somewhat tighter than that used in the $B \rightarrow K^{*} \gamma$ analysis as the expected $S / B$ is much smaller. As in the $B \rightarrow K^{*} \gamma$ analysis the dominant backgrounds come from non-resonant $e^{+} e^{-} \rightarrow q \bar{q}$ production. The $q \bar{q}$ background is suppressed by requiring $\left|\cos \theta_{\mathrm{T}}^{*}\right|<0.57$ and $\left|\cos \theta_{B}\right|<0.81$. The $\Delta E$ vs. $m_{\mathrm{ES}}$ plane is shown in Figure 6. There is one event in the signal region. The expected background is 0.9 events based on extrapolations from the sideband. We place an upper limit at $90 \%$ C.L. assuming the observed event is signal, giving

$$
\mathcal{B}\left(B^{0} \rightarrow \gamma \gamma\right)<1.7 \times 10^{-6} .
$$

This result is a substantial improvement over previous measurements [8].

\section{SEARCH FOR $B \rightarrow K^{(*)} \ell^{+} \ell^{-}$}

The decays $B \rightarrow K \ell^{+} \ell^{-}$and $B \rightarrow K^{*} \ell^{+} \ell^{-}$are expected to have branching fractions of order $(0.3-0.6) \times 10^{-6}$ and $(1-2.5) \times 10^{-6}$ respectively $[3,9,10,11]$. Figure 7 shows quark level diagrams contributing to these decays. In this analysis we searched for the following final states: $B^{+} \rightarrow K^{+} \ell^{+} \ell^{-}, B^{0} \rightarrow K_{S}^{0} \ell^{+} \ell^{-}, B^{+} \rightarrow K^{*+} \ell^{+} \ell^{-}$, and $B^{0} \rightarrow K^{0 *} \ell^{+} \ell^{-}$; where $K^{*+} \rightarrow K_{S}^{0} \pi^{+}, K^{* 0} \rightarrow K^{+} \pi^{-}$, and $\ell$ is either an electron or muon. This analysis was also carried out as a blind analysis; the signal region, as well as the near sideband, in $\Delta E$ vs. $m_{\mathrm{ES}}$ was blind during the optimization of the event selection criteria. The signal yield is extracted using an extended maximum likelihood fit in the $\Delta E$ versus $m_{\mathrm{ES}}$ plane.

Candidate events are reconstructed using electrons with a laboratory frame momentum greater than $0.5 \mathrm{GeV} / c$ and muons with a momentum greater than $1.0 \mathrm{GeV} / c$. Charged kaon candidates are required to be positively identified using the DIRC, the BABAR Cherenkov particle identification detector, and $d E / d x$ in the drift chamber and silicon vertex tracker. Our $K^{*}$ candidates are required to have a mass, $m_{K \pi}$, within 75 $\mathrm{MeV} / \mathrm{c}^{2}$ of the mean $K^{*}(892)$ mass. 


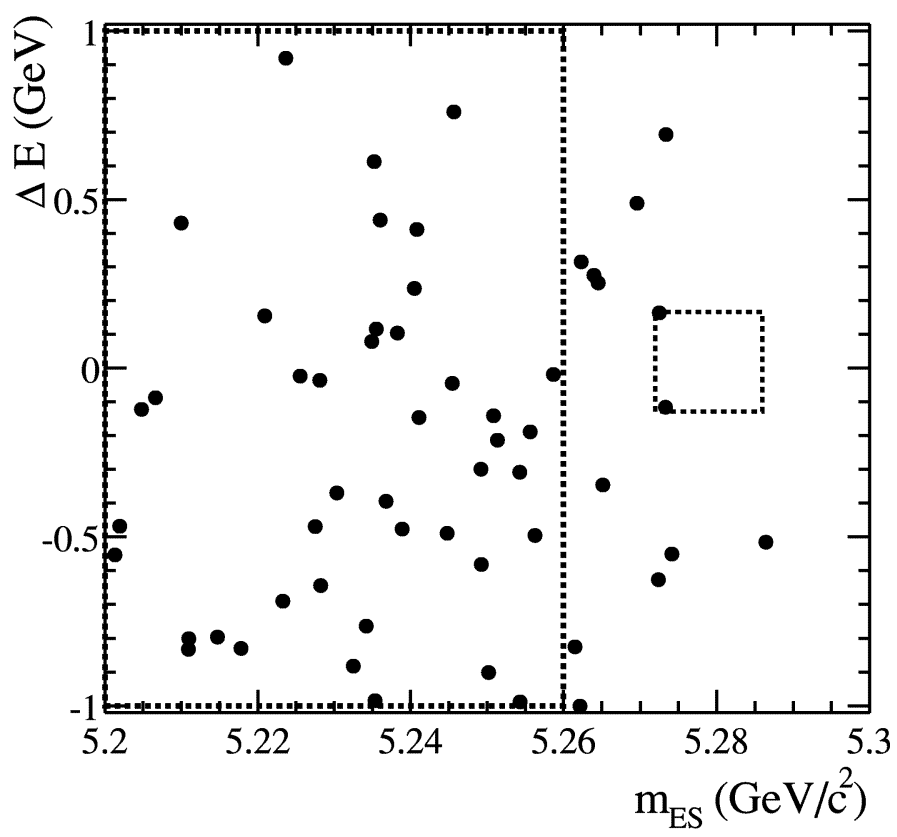

FIGURE 6. The unblinded signal region in the $B^{0} \rightarrow \gamma \gamma$ analysis. There is one event in the signal regions; consistent with the background expectation of 0.9 . events.
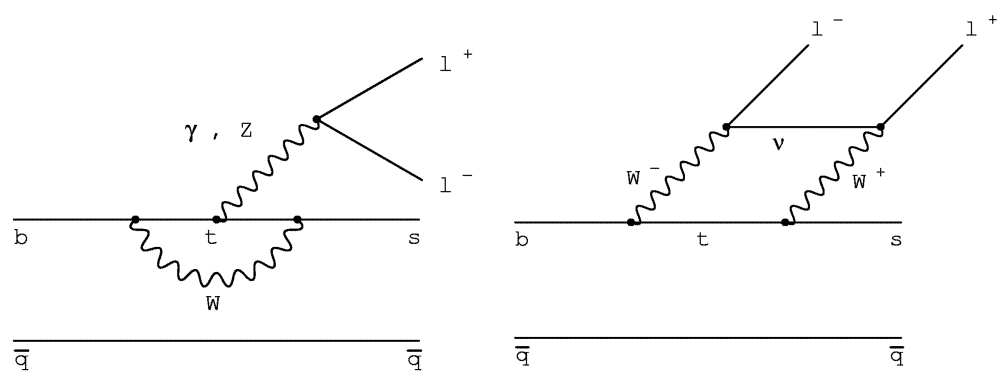

FIGURE 7. Quark level diagrams contributing to $B \rightarrow K^{(*)} \ell^{+} \ell^{-}$.

There are many backgrounds that have to be consider in this analysis. First, we have backgrounds that peak in the signal region. Most obviously, there is the $B \rightarrow J / \psi K^{(*)}$ and $B \rightarrow \psi(2 S) K^{(*)}$ modes where the charmonium states decays to leptons. These decays have the same topology as the signal and are vetoed by a correlated cut in the dilepton mass and $\Delta E$ as illustrated in Figure 8 . We also explicitly veto events in modes with a $K^{*}$ where an additional random pion, from the other $B$ decay, has been picked up to 


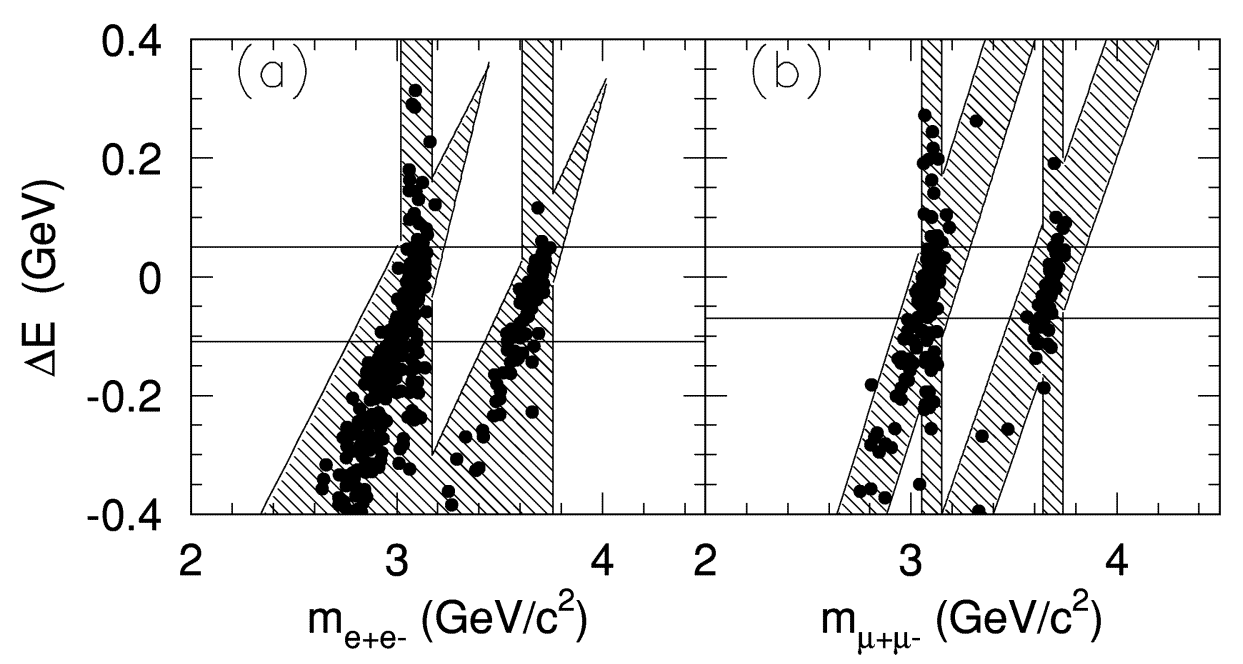

FIGURE 8. The charmonium vetoes in $B \rightarrow K^{(*)} \ell^{+} \ell^{-}$. We use a correlated cut in the dilepton mass, $m_{\ell \ell}$, and $\Delta E$ to remove the charmonium events not only in the signal region, but also in the $\Delta E$ sideband. This simplifies the description of the background shape in $\Delta E$ as otherwise there would be a large contamination from the radiative $B \rightarrow(J \psi, \psi(2 S)) K^{(*)}$ decays at low values of $\Delta E$.

compensate for the energy lost by radiation of a lepton in a $B \rightarrow(J / \psi, \psi(2 S)) K$ decay.

Besides combinatorial backgrounds in non-resonant $q \bar{q}$ events, this analysis also have a substantial combinatorial background from $B \bar{B}$ events. These background sources are suppressed by cutting on two variables that are designed to suppress the combinatorial backgrounds from continuum and $B \bar{B}$ events respectively.

The backgrounds from continuum $e^{+} e^{-} \rightarrow q \bar{q}$ production are suppressed by a Fisher discriminant which combines $\left|\cos \theta_{\mathrm{T}}^{*}\right|,\left|\cos \theta_{B}\right|, R_{2}$, and $m_{K \ell} . R_{2}$ is the ratio of second to zeroth Fox-Wolfram moment. The variable $m_{K \ell}$ uses the lepton with the charge consistent to be from a $D$ decay with the strangeness given by the $K^{(*)}$. In decays of $D$ mesons $m_{K \ell}$ will be below the $D$ mass and hence provide useful separation between signal and background.

The combinatorial background from $B \bar{B}$ decays is suppressed using a likelihood which combines several variables. The most important variable is the missing energy in the event. Leptons in $B \bar{B}$ events are typically produced in semileptonic decays and hence have a large missing energy due to the undetected neutrinos in the event. We also use information from vertexing; if we pick up candidates from different $B$ meson decays they do not come from a common vertex point and can be used to reject the $B \bar{B}$ background.

Figure 9 shows the unblinded $\Delta E$ versus $m_{\mathrm{ES}}$ plane and Fig. 10 shows the fit in the $m_{\mathrm{ES}}$ projections. The result of the fits is summarized in Table 2 . The table also includes the results of the lepton-family-number violating modes.

Combining electron and muon channels we obtain

$$
\left.\mathcal{B}\left(B \rightarrow K \ell^{+} \ell^{-}\right)=(0.0 \pm 0.3 \text { (stat. })\right) \times 10^{-6},
$$



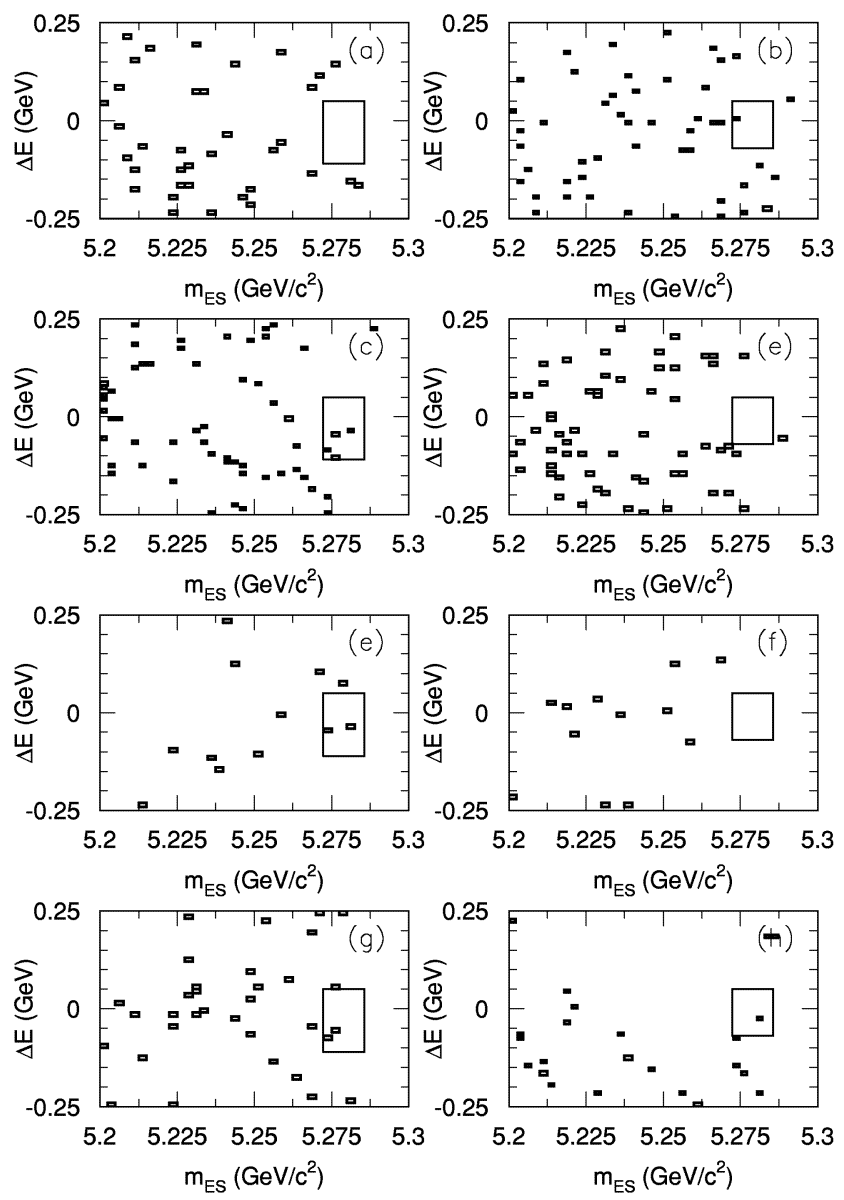

FIGURE 9. The signal region in the $\Delta E$ versus $m_{\mathrm{ES}}$ plane is shown in each of the modes. The region indicated by a box shows where the signal is expected. There is no evidence for a signal. The signal yield is extracted using a likelihood fit in the $\Delta E-m_{\mathrm{ES}}$ regions shown in these plots.

$$
\left.\mathcal{B}\left(B \rightarrow K^{*} \ell^{+} \ell^{-}\right)=(0.7 \pm 1.1 \text { (stat. })\right) \times 10^{-6} .
$$

When combining the $B \rightarrow K^{*} e^{+} e^{-}$and $B \rightarrow K^{*} \mu^{+} \mu^{-}$modes we use the constraint $\mathcal{B}\left(B \rightarrow K^{*} e^{+} e^{-}\right) / \mathcal{B}\left(B \rightarrow K^{*} \mu^{+} \mu^{-}\right)=1.2$ as given by Ref. [3]. As there is no evidence for a signal we set the upper limits

$$
\begin{gathered}
\mathcal{B}\left(B \rightarrow K \ell^{+} \ell^{-}\right)<0.6 \times 10^{-6}, \\
\mathcal{B}\left(B \rightarrow K^{*} \ell^{+} \ell^{-}\right)<2.5 \times 10^{-6} .
\end{gathered}
$$

These limits are now at the level of the predictions based on the Standard Model. Belle [12] claims a signal for $B \rightarrow K \ell^{+} \ell^{-}$with a branching fraction $\mathcal{B}\left(B \rightarrow K \ell^{+} \ell^{-}\right)=$ 


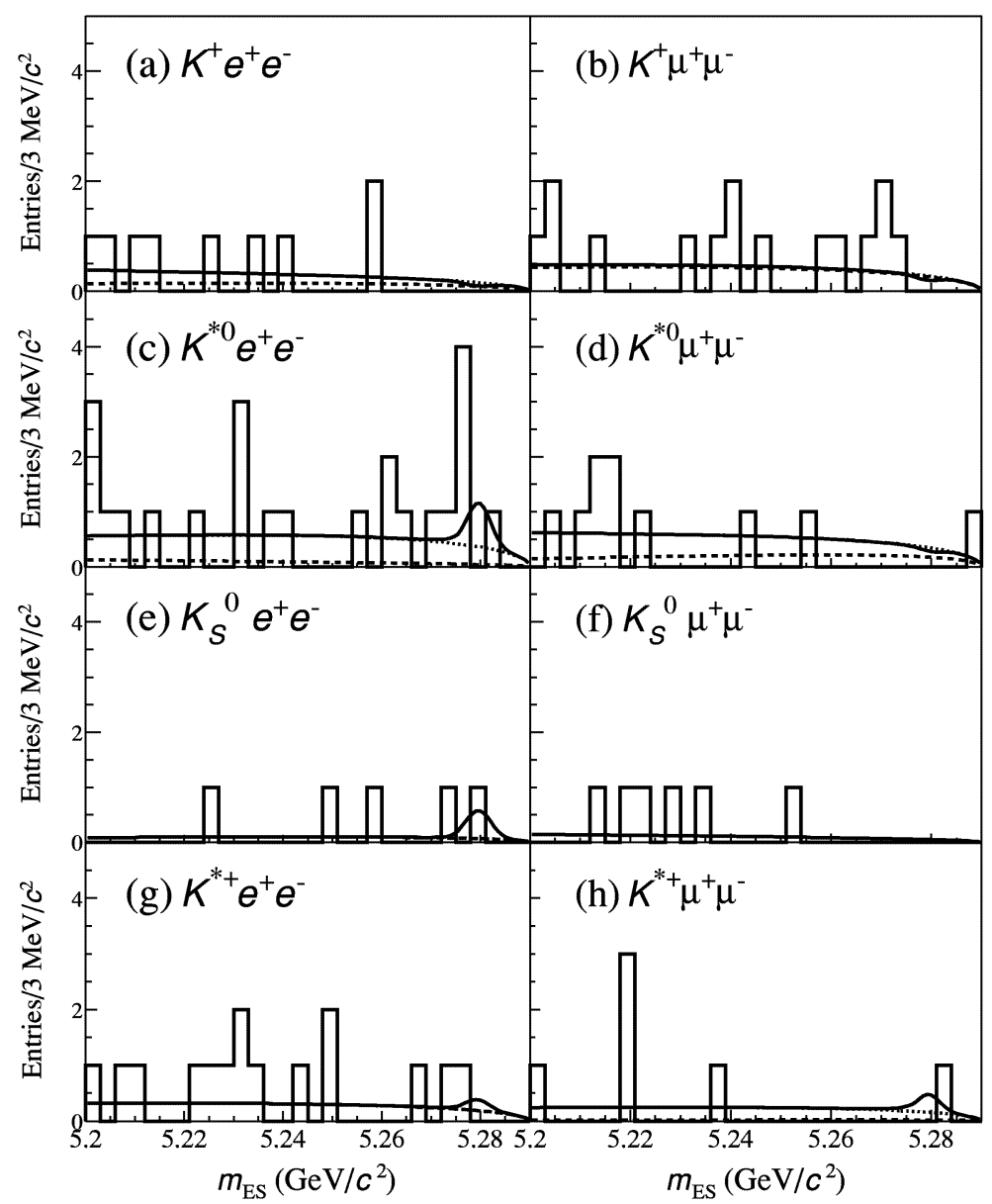

FIGURE 10. The fit is shown in an $m_{\mathrm{ES}}$ projection with $-0.11<\Delta E<0.05 \mathrm{GeV}$ for electrons and $-0.07<\Delta E<0.05 \mathrm{GeV}$ for muons.

$\left(0.75_{-0.25}^{+0.25}\right.$ (stat.) \pm 0.09 (syst. $\left.)\right) \times 10^{-6}$, which is larger than our $90 \%$ C.L. limits. Future analysis of larger data samples will soon be able to settle the issue.

\section{CONCLUSIONS}

We have reported preliminary results on a first set of measurements from BABAR in electro-weak penguin decays. All results presented here provides significant improvements over previously published results. At the time of the completion of this contribution to the conference proceedings BABAR has a data sample that is almost three times the size used for the analysis presented here and by the summer of 2002 we expect to 
TABLE 2. The results for the fits in the individual modes for $B \rightarrow K^{(*)} \ell^{+} \ell^{-}$and the leptonfamily-number violating decays $B \rightarrow K^{(*)} e^{ \pm} \mu^{\mp}$. From left to right the columns are the signal yield; the signal efficiency, excluding branching fractions for $K^{*}$ and $K$ decays; systematic uncertainties on the efficiency and fit; and the upper limit including systematic uncertainties.

\begin{tabular}{lccccc}
\hline \multicolumn{1}{c}{ Mode } & Signal yield & $\varepsilon(\%)$ & $(\Delta \mathcal{B} / \mathcal{B})_{\varepsilon}$ & $(\Delta \mathcal{B} / \mathcal{B})_{\text {fit }}$ & $\mathcal{B} / 10^{-6}$ at $90 \%$ C.L. \\
\hline$B^{+} \rightarrow K^{+} e^{+} e^{-}$ & -0.2 & 17.5 & \pm 8.6 & \pm 10.6 & 0.9 \\
$B^{+} \rightarrow K^{+} \mu^{+} \mu^{-}$ & -0.2 & 10.5 & \pm 8.6 & \pm 10.6 & 1.3 \\
$B^{0} \rightarrow K^{* 0} e^{+} e^{-}$ & 2.5 & 10.2 & \pm 10.5 & \pm 10.6 & 5.0 \\
$B^{0} \rightarrow K^{* 0} \mu^{+} \mu^{-}$ & -0.3 & 8.0 & \pm 10.8 & \pm 10.6 & 3.6 \\
$B^{0} \rightarrow K^{0} e^{+} e^{-}$ & 1.3 & 15.7 & \pm 9.3 & \pm 10.6 & 4.7 \\
$B^{0} \rightarrow K^{0} \mu^{+} \mu^{-}$ & 0.0 & 9.6 & \pm 11.4 & \pm 10.6 & 4.5 \\
$B^{+} \rightarrow K^{*+} e^{+} e^{-}$ & 0.1 & 8.5 & \pm 11.4 & \pm 10.6 & 10.0 \\
$B^{+} \rightarrow K^{*+} \mu^{+} \mu^{-}$ & 1.0 & 5.8 & \pm 12.0 & \pm 10.6 & 17.5 \\
\hline$B^{+} \rightarrow K^{+} e^{ \pm} \mu^{\mp}$ & -0.6 & 16.8 & \pm 8.6 & \pm 10.6 & 1.0 \\
$B^{0} \rightarrow K^{* 0} e^{ \pm} \mu^{\mp}$ & 0.6 & 11.9 & \pm 10.6 & \pm 10.6 & 2.7 \\
$B^{0} \rightarrow K^{0} e^{ \pm} \mu^{\mp}$ & 0.8 & 14.6 & \pm 10.3 & \pm 10.6 & 3.3 \\
$B^{+} \rightarrow K^{*+} e^{ \pm} \mu^{\mp}$ & -0.4 & 9.3 & \pm 11.7 & \pm 10.6 & 8.7 \\
\hline
\end{tabular}

have a sample of $100 \mathrm{fb}^{-1}$. Hence, in the near future we should expect many of the results presented here to be updated.

\section{ACKNOWLEDGMENTS}

I would like to thank all my colleagues at BABAR and PEP-II for making it possible to present these results.

\section{REFERENCES}

1. R. Ammar et al. (CLEO Collaboration), Phys. Rev. Lett., 71, 674 (1993).

2. M.S. Alam et al. (CLEO Collaboration), Phys. Rev. Lett., 74, 2885 (1995).

3. A. Ali, et al., Phys. Rev., D61, 074024 (2000).

4. B. Aubert et al. (BABAR Collaboration), submitted to Nucl. Instrum. Meth.

5. S.W. Bosch and G. Buchalla, hep-ph/0106081.

6. M. Beneke et al., hep-ph/0106067.

7. G.G. Devidze and G.R. Jibuti, Phys. Lett., B429, 48 (1998).

8. D.E. Groom, et al., Eur. Phys. J., C15, 1 (2000).

9. P. Colangelo, et al., Phys. Rev., D53, 3672 (1996).

10. D. Melikhov, N. Nikitin, and S. Simula, Phys. Rev., D57, 6814 (1998).

11. T.M. Aliev et al., Phys. Lett., B400, 194 (1997).

12. M. Nakao, in these proceedings. 